\section{A Jerk Model for Tracking Highly Maneuvering Targets}

KISHORE MEHROTRA

PRAVAS R. MAHAPATRA

Indian Institute of Science

A model of target motion in three-dimensional space that includes position derivatives up to the third order is developed. Compared with available models, which include terms at the most up to the second derivative, the model introduced in this work, called the jerk model, can more accurately describe agile target maneuvers which are likely to contain significant higher order derivatives. A compatible 4-state Kalman filter to perform tracking in conjunction with the jerk model is also presented, and an initialization procedure for the filter is provided. The improved performance of the jerk model over a lower order model is illustrated through numerical simulation.

Manuscript received November 12, 1994; revised February 28, 1996.

IEEE Log No. T-AES/33/4/06834.

Authors' current addresses: K. Mehrotra, 33 Halliwell Avenue, Papanui, Christchurch, New Zealand; P. R. Mahapatra, Dept. of Aerospace Engineering, Indian Institute of Science, Bangalore 560 012, India.

0018-9251/97/\$10.00 (c) 1997 IEEE

\section{INTRODUCTION}

Mathematical modeling of the target tracking process has been a topic of extensive study. The alpha-beta and the alpha-beta-gamma trackers [1-31 represent the classical approaches to this problem. Since the early sixties the Kalman filter and its variants have emerged as the preferred filters for tracking applications. In this approach the state of the tracked object consists of its position and the time-derivatives of displacement. The displacement of an arbitrarily maneuvering target would, in general, have a number of non-zero derivatives. An accurate model of the target motion should ideally include all these derivatives. However, since a large number of derivatives make a model difficult to implement, commonly used target models take into account only a few derivatives of the target displacement. The target motion models of the highest order currently available in open literature include terms up to the target acceleration, i.e., the second derivative of the target position. Such models are referred to as acceleration models in this work.

In modern tracking analysis, the target kinematics are assumed to be the result of a forcing function. Unfortunately, there is no direct and unique way of obtaining the forcing function, and frequent recourse has been taken to an intuitive approach towards modeling this function. This has led to a wide diversity of analytical formulations of the tracking problem (e.g. [4-8]). Particular mention may be made of the model by Singer [5] which is quite successful in modeling the tracking of maneuvering targets in a wide variety of situations involving different types of filters. A different approach by Bar-Shalom and Birmiwal [9] does not rely on a statistical description of the maneuver as a random process, but introduces extra state components in the state model when maneuver is detected.

There are many types of target motion, especially those involving the modern generation of highly maneuvering aerospace vehicles, which call for better tracking performance than what is provided by acceleration models. The reason for the inadequate tracking performance of current models is that the higher order derivatives in the case of very highly maneuvering targets are not insignificant, leading to model inaccuracies when terms only up to the second order are included.

In this work, a solution to the problem of model order insufficiency is sought by including one more derivative in the target model. Ther term is the third derivative of the target position, i.e., the acceleration rate orjerk of the target. Accordingly, the model developed here is referred to as ajerk model of the target motion. Recently jerk models have been used in the context of fixed-gain alpha-beta-gamma trackers $[10,11]$. The current paper provides a full 4 -state 
Kalman filter tracking analysis employing the jerk model.

\section{GAUSS-MARKOV MODELING OF TARGET MOTION INCLUDINGJERK}

Following Singer [5], we start with the target jerk as a process which is correlated in time. This means that if a target experiences a certain acceleration rate at a time $t$ then it is likely to be experiencing the same jerk also at the time instant $t+\tau$ for sufficiently small $\tau$. Relatively slow or lazy maneuvers will give rise to correlated jerk inputs for longer time scales than fast and evasive ones which correspond to a faster decay of jerk correlation. An exponential correlation function is assumed to represent the target jerk:

$$
\begin{aligned}
r_{j}(\tau) & =E\{j(t) j(t+\tau)\} \\
& =\sigma_{j}^{2} \exp (-\alpha|\tau|)
\end{aligned}
$$

where $\sigma_{j}^{2}$ is the variance of the target jerk and Q is the reciprocal of the jerk time constant, and $E\{\cdot\}$ denotes expectation.

The Wiener-Kolmogorov whitening procedure is used on $r_{j}(\tau)$ to represent the jerk $j(t)$ as a function of time, driven by a white noise input $w(t)$. The Laplace transform of the jerk correlation function $r_{j}(\tau)$ is

$$
\begin{aligned}
R(s) & =L\left\{r_{j}(\tau)\right\}=\frac{-2 \alpha \sigma_{j}^{2}}{(s-a)(s+a)} \\
& =H(s) H(-s) W(s)
\end{aligned}
$$

where

$$
H(s)=1 /(s+\alpha)
$$

and

$$
W(s)=2 \alpha \sigma_{j}^{2} .
$$

The quantity $H(s)$ is the transfer function of the whitening filter for the jerk $j(t)$, and $W(s)$ is the transform of the white noise $w(t)$ that drives $j(t)$. The differential equation that results from (3) is

$$
j(t)=-\alpha j(t)+w(t) .
$$

The autocorrelation function $r_{w}(\tau)$ of the white noise input is found by taking the inverse Laplace transform of (4) as

$$
\begin{aligned}
r_{w}(\tau) & =2 \alpha \sigma_{j}^{2} \delta(\tau) \\
& =Q, \delta(\tau)
\end{aligned}
$$

where $\delta(\tau)$ is the unit impulse function or "delta function" located at the origin, and $Q,=2 \alpha \sigma_{j}^{2}$ is the variance of $w(t)$.

A brief discussion of the parameters of the jerk description (5) is in order. As discussed in the first paragraph of this section, the correlation parameter $\alpha$ permits the modeling of different classes of targets: small Q for targets with sustained jerk levels, and high Q for targets with rapidly fluctuating jerk. The white noise component of the jerk model permits the target to superimpose random jerk components over its sustainedjerk capabilities. In effect, the value of $\alpha$ serves to adjust the model order to fractional values, with a very low $\alpha$ corresponding to the third-order (jerk) model, and a very high Q making the model behave like a second-order (constant acceleration) model.

The state equation ( 5 ) for the continuous-time jerk model can be written in a state space framework as

$$
\frac{d}{d t}\left[\begin{array}{l}
x \\
\dot{x} \\
\ddot{x} \\
\dddot{x}
\end{array}\right]=\left[\begin{array}{rrrr}
0 & 1 & 0 & 0 \\
0 & 0 & 1 & 0 \\
0 & 0 & 0 & 1 \\
0 & 0 & 0 & -\alpha
\end{array}\right]\left[\begin{array}{l}
x \\
\dot{x} \\
\ddot{x} \\
\dddot{x}
\end{array}\right]+\left[\begin{array}{l}
0 \\
0 \\
0 \\
1
\end{array}\right] w(t)
$$

where $\mathbf{x}, \dot{x}, \ddot{x}$, and $\dddot{x}$ denote, respectively, the position, velocity, acceleration, and jerk of the target. The equation is of the standard form

$$
\mathbf{X}=\mathbf{A X}+\mathbf{B} w(t)
$$

where the meanings of the vector and matrix symbols are clear by comparison with (7). The measurement vector at the $(k+1)$ th instant is

$$
\mathbf{Z}(k+1)=\mathbf{H X}(k+1)+\mathbf{V}(k+1)
$$

where $\mathbf{H}$ is the system measurement or observation matrix, and $\mathbf{V}$ is the measurement noise vector, assumed uncorrelated to the process noise.

Using the jerk model for target motion evolved in this section, we now proceed to analyze the tracking problem using a compatible Kalman filter.

\section{TARGET TRACKING IN ONE DIMENSION}

We first consider target motion in one dimension to help visualize the tracking process better. Equations for tracking in three dimensions follow in a later section. A discrete Kalman filter is used for tracking a target with motion modeled as per (7). Discretization of (7) yields

$$
\mathrm{X}(\mathrm{k}+1)=\mathbf{F}(k+1, k) \mathbf{X}(k)+\mathbf{u}(k)
$$

where

$$
\mathbf{F}(k+1, k)=e^{\mathbf{A}\left(t_{k+1}-t_{k}\right)}
$$

is the state transition matrix of the linear time-

invariant system between the instants $k$ and $k+1$, and $\mathbf{u}(k)$ is a discrete white noise vector defined as

$$
\mathbf{u}(k)=\int_{t_{k}}^{t_{k+1}} \mathbf{F}\left(t_{k+1}, \tau\right) \mathbf{B}(\tau) w(\tau) d \tau
$$

The variance $\mathbf{Q}(k)$ of the process noise $u(k)$ is

$$
\begin{aligned}
\mathbf{Q}(k)= & E\left\{\mathbf{u}(k) \mathbf{u}^{T}(k)\right\} \\
= & \int_{t_{k}}^{t_{k+1}} \int_{t_{k}}^{t_{k+1}} \mathbf{F}\left(t_{k+1}, \tau\right) \mathbf{B}(\tau) E\left\{w(\tau) w^{T}(\nu)\right\} \\
& x \mathbf{B}^{T}(\nu) \mathbf{F}^{T}\left(t_{k+1}, \nu\right) d \tau d \nu .
\end{aligned}
$$


The state transition matrix is obtained through a series expansion of the right-hand side of (11), and substitution of the matrix A. This yields

$$
\mathbf{F}(T)=\left[\begin{array}{cccc}
1 & T & T^{2} / 2 & p_{1} \\
0 & 1 & T & q_{1} \\
0 & 0 & 1 & r_{1} \\
\text { to } & 0 & 0 & s_{1}
\end{array}\right]
$$

where

$$
\begin{aligned}
T & =t_{k+1}-t_{k} \\
p_{1} & =\frac{T^{3}}{3 !}-\frac{\alpha T^{4}}{4 !}+\frac{\alpha^{2} T^{5}}{5 !}-\cdots \\
& =\left(2-2 \alpha T+\alpha^{2} T^{2}-2 e^{-\alpha T}\right) /\left(2 \alpha^{3}\right) \\
q_{1} & =\frac{T^{2}}{2 !}-\frac{\alpha T^{3}}{3 !}+\frac{\alpha^{2} T^{4}}{4 !}-. \\
& =\left(e^{-\alpha T}-1+\alpha T\right) / \alpha^{2} \\
r_{1} & =T-\frac{\alpha T^{2}}{2 !}+\frac{\alpha^{2} T^{3}}{3 !}-\frac{\alpha^{3} T^{4}}{4 !}+\cdots \\
& =\left(1-e^{-\alpha T}\right) / \alpha \\
s_{1} & =1-\alpha T+\frac{\alpha^{2} T^{2}}{2 !}-\frac{\alpha^{3} T^{3}}{3 !}+\frac{\alpha^{4} T^{4}}{4 !}- \\
& -e^{-\alpha T}
\end{aligned}
$$

For the special case in which the product $\alpha T$ is small, the following simpler form of $\mathbf{F}(T)$ results

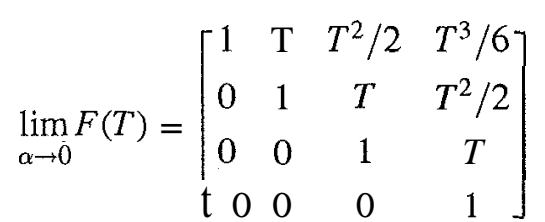

which means that the filter assumes the jerk to be constant between the sampling instants.

In (13), the expectation $E\left\{w(\tau) w^{T}(\nu)\right\}$ is the same as the autocorrelation $r_{w}(\tau)$ in (6). Also, for the linear time-invariant system, $\mathbf{F}\left(t_{k+1}, \tau\right)=\mathbf{F}\left(t_{k+1}-\tau\right)$. Using these facts in (13),

$$
\mathbf{Q}(k)=Q_{w} \int_{t_{k}}^{t_{k+1}} \mathbf{F}\left(t_{k+1}-\tau\right) \mathbf{B}(\tau) \mathbf{B}^{T}(\tau) \mathbf{F}^{T}\left(t_{k+1}-\tau\right) d \tau .
$$

Substituting the expanded form of $\mathrm{B}$, and the matrix $\mathrm{F}$ from (14),

$$
\mathbf{Q}(k)=Q_{w}\left[\begin{array}{cccc}
\int p_{1}^{2} d \tau & \int p_{1} q_{1} d \tau & \int p_{1} r_{1} d \tau & \int p_{1} s_{1} d \tau \\
\int p_{1} q_{1} d \tau & \int q_{1}^{2} d \tau & \int q_{1} r_{1} d \tau & \int q_{1} s_{1} d \tau \\
\int p_{1} r_{1} d \tau & \int q_{1} r_{1} d \tau & \int r_{1}^{2} d \tau & \int r_{1} s_{1} d \tau \\
\int p_{1} s_{1} d \tau & \int q_{1} s_{1} d \tau & \int r_{1} s_{1} d \tau & \int s_{1}^{2} d \tau
\end{array}\right]
$$

where each of the quantities $p_{1}, q_{1}, r_{1}$ and $s_{1}$ is evaluated at the instant $\left(t_{k+1}-\tau\right)$. Substituting the

value of $\mathrm{Q}$, from (6),

$$
\left.\mathbf{Q}(k)=2 \alpha \sigma_{j}^{2} \mid \begin{array}{llll}
q_{11} & q_{12} & q_{13} & q_{14} \\
q_{21} & q_{22} & q_{23} & q_{24} \\
q_{31} & q_{32} & q_{33} & q_{34} \\
q_{41} & q_{42} & q_{43} & q_{44}
\end{array}\right]
$$

where the elements of the symmetric matrix are

$$
\begin{aligned}
q_{11}= & \int p_{1}^{2}\left(t_{k+1}-u\right) d u \\
= & \frac{1}{2 \alpha^{7}}\left(\frac{\alpha^{5} T^{5}}{10}-\frac{\alpha^{4} T^{4}}{2}+\frac{4 \alpha^{3} T^{3}}{3}-2 \alpha^{2} T^{2}+2 \alpha T-3\right. \\
& \left.\quad+4 e^{-\alpha T}+2 \alpha^{2} T^{2} e^{-\alpha T}-e^{-2 \alpha T}\right) \\
q_{12}= & q_{21}=\int p_{1}\left(t_{k+1}-u\right) q_{1}\left(t_{k+1}-u\right) d u \\
= & \frac{1}{2 a 6}\left(1-2 a T+2 \alpha^{2} T^{2}-\alpha^{3} T^{3}+\frac{\alpha^{4} T^{4}}{4}\right. \\
& \left.\quad+e^{-2 \alpha T}+2 \alpha T e^{-\alpha T}-2 e^{-\alpha T}-\alpha^{2} T^{2} e^{-\alpha T}\right) \\
q_{13}= & q_{31}=\int p_{1}\left(t_{k+1}-u\right) r_{1}\left(t_{k+1}-u\right) d u \\
= & +\left(2 \alpha T-\alpha^{2} T^{2}+\alpha^{3} T^{3}-3-e^{-2 \alpha T}\right. \\
& 2 a 5 \\
q_{44}= & \left.\quad+4 e^{-\alpha T}+\alpha^{2} T^{2} e^{-\alpha T}\right) \\
= & (1 / 2 \alpha)\left(1-e^{-2 \alpha T}\right) . \\
q_{34}^{2}\left(t_{k+1}-u\right) d u & q_{43}=\int r_{1}\left(t_{k+1}-u\right) s_{1}\left(t_{k+1}-u\right) d u \\
= & \left(1 / 2 \alpha^{2}\right)\left(1-2 e^{-\alpha T}+e^{-2 \alpha T}\right) \\
q_{14}= & q_{41}=\int p_{1}\left(t_{k+1}-u\right) s_{1}\left(t_{k+1}-u\right) d u \\
= & \left(1 / 2 \alpha^{4}\right)\left(1+e^{-2 \alpha T}-2 e^{-\alpha T}-\alpha^{2} T^{2} e^{-\alpha T}\right) \\
q_{24}= & q_{42}=\int q_{1}\left(t_{k+1}-u\right) s_{1}\left(t_{k+1}-u\right) d u \\
= & \int q_{1}^{2}\left(t_{k+1}-u\right) d u \\
= & \frac{1}{2 \alpha^{5}}\left(1-e^{-2 \alpha T}+\frac{2 \alpha^{3} T^{3}}{3}+2 \alpha T-2 \alpha^{2} T^{2}-4 \alpha T e^{-\alpha T}\right) \\
q_{23}= & q_{32}=\int q_{1}\left(t_{k+1}-u\right) r_{1}\left(t_{k+1}-u\right) d u \\
= & \left(1 / 2 \alpha^{4}\right)\left(1+\alpha^{2} T^{2}-2 \alpha T+2 \alpha T e^{-\alpha T}+e^{-2 \alpha T}-2 e^{-\alpha T}\right)
\end{aligned}
$$


When $\alpha T$ is sufficiently small the covariance matrix $\mathbf{Q}(k)$ reduces to

$$
\lim _{\alpha T \rightarrow 0} \mathbf{Q}(k)=2 \alpha \sigma_{j}^{2}\left[\begin{array}{cccc}
T^{7} / 252 & T^{6} / 72 & T^{5} / 30 & T^{4} / 24 \\
T^{6} / 72 & T^{5} / 20 & T^{4} / 8 & T^{3} / 6 \\
T^{5} / 30 & T^{4} / 8 & T^{3} / 3 & T^{2} / 2 \\
T^{4} / 24 & T^{3} / 6 & T^{2} / 2 & T
\end{array}\right]
$$

which can be used in conjunction with the expression (16)for $\mathrm{F}(T)$ to simplify computations.

For a fixed sampling rate, as $a \rightarrow \infty$ the jerk model is reduced to a constant acceleration filter: the state transition matrix becomes

$$
\lim _{\alpha \rightarrow \infty} \mathrm{F}(T)=\left[\begin{array}{cccc}
1 & T & T^{2} / 2 & 0 \\
0 & 1 & T & 0 \\
0 & 0 & 1 & 0
\end{array}\right]
$$

and the process noise covariance matrix is

$$
\operatorname{dimb} \mathbf{Q}(k)=\left[\begin{array}{cccc}
0 & 0 & 0 & 0 \\
0 & 0 & \emptyset & 0 \\
0 & 0 & 0 & 0 \\
0 & 0 & 0 & \sigma_{j}^{2}
\end{array}\right]
$$

\section{INITIALIZATION OF TRACKING FILTER}

The Kalman filter is initialized by using the first three measurements to determine the position, velocity, and acceleration estimates. The initial jerk estimate is taken to be equal to zero. The relations utilized to initialize the state variables are

$$
\begin{aligned}
& \hat{x}(3)=M_{x}(3) \\
& \hat{\dot{x}}(3)=\left[M_{x}(3)-M_{x}(2)\right] / T \\
& \hat{x}(3)=\left[M_{x}(3)-2 M_{x}(2)+M_{x}(1)\right] / T^{2}
\end{aligned}
$$

and

$$
\dddot{x}(3)=0 \text {. }
$$

The first three measurements $M_{x}(1), M_{x}(2)$, and $M_{x}(3)$ are the samples of the true positions of the target, corrupted by measurement noise,

$$
\begin{aligned}
& M_{x}(1)=x(1)+\nu(1) \\
& M_{x}(2)=x(2)+\nu(2) \\
& M_{x}(3)=x(3)+\nu(3) .
\end{aligned}
$$

We now derive the expressions for the covariance components which are initialized by (22). Let $u_{1}(k)$, $u_{2}(k), u_{3}(k)$, and $u_{4}(k)$ be the components of process noise driving the state equation in the discrete model as given in (10).Let the errors in position, velocity, acceleration, and jerk estimates be denoted by $\varepsilon_{1}, \varepsilon_{2}$,

$\varepsilon_{3}$, and $\varepsilon_{4}$ respectively. Then, using the relations (22) and (23),

$$
\begin{aligned}
& \varepsilon_{1}(3 \mid 3)=x(3)-\hat{x}(3 \mid 3) \\
& =x(3)-x(3)-\nu(3) \\
& =-\nu(3) \\
& \varepsilon_{2}(3 \mid 3)=\dot{x}(3)-\hat{\dot{x}}(3 \mid 3) \\
& =\dot{x}(3)-\left[M_{x}(3)-M_{x}(2)\right] / T \\
& =\dot{x}(3)-[x(3)+\nu(3)-x(2)-\nu(2)] / T \\
& =\dot{x}(3)-\left[x(2)+T \dot{x}(2)+\left(T^{2} / 2\right) \ddot{x}(2)+p_{1} \ddot{x}(2)\right. \\
& \left.+\nu(3)-x(2)-\nu(2)+u_{1}(2)\right] / T \\
& =\dot{x}(2)+T \ddot{x}(2)+q_{1} \ddot{x}(2)+u_{2}(2) \\
& -\left[T \dot{x}(2)+\left(T^{2} / 2\right) \ddot{x}(2)+p_{1} \ddot{x}(2)\right. \\
& \left.+u_{1}(2)+\nu(3)-\nu(2)\right] / T \\
& =(T / 2) \ddot{x}(2)+\left(q_{1}-p_{1} / T\right) \ddot{x}(2)+u_{2}(2) \\
& -u_{1}(2) / T-\nu(3) / T+\nu(2) / T \\
& =(T / 2)\left[\ddot{x}(1)+r_{1} \ddot{x}(1)+u_{3}(1)\right] \\
& +\left(q_{1}-p_{1} / T\right)\left[s_{1} \dddot{x}(1)+u_{4}(1)\right] \\
& +u_{2}(2)-u_{1}(2) / T-\nu(3) / T+\nu(2) / T \\
& =(T / 2) \ddot{x}(1)+\left(q_{1} s_{1}-p_{1} s_{1} / T+r_{1} T / 2\right) \ddot{x}(1) \\
& +\left(q_{1}-p_{1} / T\right) u_{4}(1)+(T / 2) u_{3}(1)+u_{2}(2)-u_{1}(2) / T \\
& -\nu(3) / T+\nu(2) / T \\
& \varepsilon_{3}(3 \mid 3)=\ddot{x}(3)-\hat{x}(3 \mid 3) \\
& =\ddot{x}(3)-\left[M_{x}(3)-2 M_{x}(2)+M_{x}(1)\right] / T^{2} \\
& =\ddot{x}(3)-[x(3)+\nu(3)-2 x(2)-2 \nu(2)+x(1)+\nu(1)] / T^{2} \\
& =\ddot{x}(2)+r_{1} \ddot{x}(2)+u_{3}(2) \\
& -\left[\begin{array}{c}
x(2)+T \dot{x}(2)+\left(T^{2} / 2\right) \ddot{x}(2)+p_{1} \ddot{x}(2)+u_{1}(2) \\
+\nu(3)-2 x(2)-2 \nu(2)+x(1)+\nu(1)
\end{array}\right] / T^{2} \\
& =\ddot{x}(2) / 2+\left(r_{1}-p_{1} / T^{2}\right) \ddot{x}(2)-\dot{x}(2) / T \\
& +[x(2)-x(1)] / T^{2}+u_{3}(2)-u_{1}(2) / T^{2} \\
& -\nu(3) / T^{2}+2 \nu(2) / T^{2}-\nu(1) / T^{2} \\
& =\left[\ddot{x}(1)+r_{1} \dddot{x}(1)+u_{3}(1)\right] / 2+\left(r_{1}-p_{1} / T^{2}\right) \\
& x\left[s_{1} \ddot{x}(1)+u_{4}(1)\right] \\
& -\left[\dot{x}(1)+T \ddot{x}(1)+q_{1} \dddot{x}(1)+u_{2}(1)\right] / T \\
& +\left[\mathbf{x}(1)+T \dot{x}(1)+\left(T^{2} / 2\right) \ddot{x}(1)\right. \\
& \left.+p_{1} \dddot{x}(1)+u_{1}(1)-x(1)\right] / T^{2} \\
& +u_{3}(2)-u_{1}(2) / T^{2}-\nu(3) / T^{2}+2 \nu(2) / T^{2}-\nu(1) / T^{2} \\
& =\left(r_{1} / 2+r_{1} s_{1}+p_{1} / T^{2}-q_{1} / T-p_{1} s_{1} / T^{2}\right) x(1) \\
& +u_{3}(1) / 2+u_{3}(2)+\left(r_{1}-p_{1} / T^{2}\right) u_{4}(1) \\
& -u_{1}(2) / T^{2}+u_{1}(1) / T^{2}-u_{2}(1) / T \\
& -\nu(1) / T^{2}-\nu(3) / T^{2}+2 \nu(2) / T^{2}
\end{aligned}
$$




$$
\begin{aligned}
\varepsilon_{4}(3 \mid 3) & =\mathrm{x}(3)-\dddot{x}(3 \mid 3) \\
& =s_{1} \dddot{x}(2)+u_{4}(2)-0 \\
& =s_{1}\left[s_{1} \dddot{x}(1)+u_{4}(1)\right]+u_{4}(2) \\
& =s_{1}^{2} \dddot{x}(1)+s_{1} u_{4}(1)+u_{4}(2) .
\end{aligned}
$$

Now, using the basic definitions, the elements of the covariance matrix corresponding to these errors are found as

$$
\begin{aligned}
p_{11}= & E\left\{\varepsilon_{1}(3 \mid 3)\right\}^{2} \\
= & E\left\{\nu^{2}(3)\right\} \\
= & \sigma_{x}^{2} \\
p_{22}= & E\left\{\varepsilon_{2}(3 / 3)\right\}^{2} \\
= & \left(T^{2} / 4\right) \sigma_{m}^{2}+\left(q_{1} s_{1}-p_{1} s_{1} / T+r_{1} T / 2\right)^{2} \sigma_{j}^{2} \\
& +\left(q_{1}-p_{1} / T\right)^{2} E\left\{u_{4}^{2}\right\}+\left(T^{2} / 4\right) E\left\{u_{3}^{2}\right\} \\
& +E\left\{u_{2}^{2}\right\}+\left(1 / T^{2}\right) E\left\{u_{1}^{2}\right\}+2 \sigma_{x}^{2} / T^{2} \\
& +T\left(q_{1}-p_{1} / T\right) E\left\{u_{3} u_{4}\right\}-(2 / T) E\left\{u_{1} u_{2}\right\} \\
p_{33}= & \left(r_{1} / 2+r_{1} s_{1}+p_{1} / T^{2}-q_{1} / T-p_{1} s_{1} / T^{2}\right)^{2} \sigma_{j}^{2} \\
& +\left(6 / T^{4}\right) \sigma_{x}^{2}+\left(2 / T^{4}\right) E\left\{u_{1}^{2}\right\} \\
& +\left(1 / T^{2}\right)\left[E\left\{u_{2}^{2}\right\}-E\left\{u_{3} u_{1}\right\}\right]+(5 / 4) E\left\{u_{3}^{2}\right\} \\
& +\left(r_{1}-p_{1} / T^{2}\right)^{2} E\left\{u_{4}^{2}\right\}-(1 / T) E\left\{u_{3} u_{2}\right\} \\
& +\left(r_{1}-p_{1} / T^{2}\right)\left[E\left\{u_{3} u_{4}\right\}+\left(2 / T^{2}\right) E\left\{u_{4} u_{1}\right\}\right. \\
& \left.\quad-(2 / T) E\left\{u_{4} u_{2}\right\}\right] \\
& -\left(2 / T^{3}\right) E\left\{u_{1} u_{2}\right\} \\
p_{34}= & s_{1}^{4} \sigma_{j}^{2}+\left(s_{1}^{2}+1\right) E\left\{u_{4}^{2}\right\} \\
& +E\left\{u_{4} u_{3}\right\}\left(1+s_{1} / 2\right)+s_{1}\left(r_{1}-p_{1} / T^{2}\right) E\left\{u_{4}^{2}\right\} \\
& +\left[\left(s_{1}-1\right) / T^{2}\right] E\left\{u_{4} u_{1}\right\}-\left(s_{1} / T\right) E\left\{u_{4} u_{2}\right\} . \\
p_{12}= & p_{21}=\sigma_{x}^{2} / T \\
p_{14}= & p_{41}=0 \\
p_{13}= & p_{31}=\sigma_{x}^{2} / T^{2} \\
p_{32}= & p_{23}=\left(q_{1} s_{1}-p_{1} s_{1} / T+r_{1} T / 2\right) \\
& +\left(r_{1} / 2+r_{1} s_{1}+p_{1} / T^{2}-q_{1} / T-p_{1} s_{1} / T^{2}\right) \sigma_{j}^{2} \\
& +3 \sigma_{x}^{2} / T^{3}+E\left\{u_{4} u_{3}\right\}\left[q_{1} / 2+r_{1} T / 2-p_{1} / T\right] \\
& +\left(r_{1}-p_{1} / T^{2}\right)\left(q_{1}-p_{1} / T\right) E\left\{u_{4}^{2}\right\} \\
& +\left(1 / T^{2}\right)\left(q_{1}-p_{1} / T\right) E\left\{u_{4} u_{1}\right\}+(T / 4) E\left\{u_{3}^{2}\right\} \\
& -(1 / T) E\left\{u_{1} u_{3}\right\}+(1 / 2) E\left\{u_{2} u_{3}\right\}-\left(1 / T^{2}\right) E\left\{u_{1} u_{2}\right\} \\
& +\left(1 / T^{3}\right) E\left\{u_{1}^{2}\right\}-\left(q_{1} / T-p_{1} / T^{2}\right) E\left\{u_{4} u_{2}\right\} \\
p_{24}= & p_{42}=s_{1}^{2}\left(q_{1} s_{1}-p_{1} s_{1} / T+r_{1} T / 2\right) E\{\ddot{x}(1)\}^{2} \\
& \left.+s_{1}-p_{1} / T\right) E\left\{u_{4}^{2}\right\} \\
&
\end{aligned}
$$

In the above set of equations, the term $E\left\{\ddot{x}^{2}(1)\right\}$ equals $\sigma_{m}^{2}$, the standard deviation of the target acceleration, and $E\left\{\dddot{x}^{2}(1)\right\}$ equals $\sigma_{j}^{2}$, the standard deviation of the target jerk. Further, the terms of the type $E\left\{u_{1} u_{2}\right\}, E\left\{u_{2} u_{3}\right\}$, etc. are the elements of the matrix $\mathbf{Q}(k)$ in (19).

When $\alpha T$ is small the covariance matrix initialization simplifies to

$$
\mathbf{P}=\left[\begin{array}{cccc}
\sigma_{x}^{2} & \mathbb{I}_{i}^{\prime \prime} / T & \sigma_{x}^{2} / T^{2} & 0 \\
\sigma_{x}^{2} / T & 2 \sigma_{x}^{2} / T^{2} & 3 \sigma_{x}^{2} / T^{3} & (5 / 6) \sigma_{j}^{2} T^{2} \\
\sigma_{x}^{2} / T^{2} & 3 \sigma_{x}^{2} / T^{3} & 6 \sigma_{x}^{2} / T^{4} & \sigma_{j}^{2} T \\
0 & (5 / 6) \sigma_{j}^{2} T^{2} & \sigma_{j}^{2} T & \sigma_{j}^{2}
\end{array}\right] .
$$

\section{TRACKING IN THREE DIMENSIONS}

We now proceed to analyze the more complex but realistic problem of tracking in 3-dimensional space. The measurements are assumed to be available in range, azimuth, and elevation $(Y, \theta, \varphi)$ dimensions. A 4-state filter having jerk as the highest order state variable is developed. For comparison purposes, a 3 -state filter with terms up to acceleration is also developed here.

The model equations for the 3-dimensional Kalman filter are written by generalizing the basic 1-dimensional equations. This is done here for the acceleration model (denoted by the subscript a) as well as the jerk model (subscript $j$ ). The equations for the acceleration and jerk models are, respectively,

$$
\begin{aligned}
& \mathbf{X}_{a}(k+1)=\mathbf{F}_{a} \mathbf{X}_{a}(k)+\mathbf{W}_{a}(k) \\
& \mathbf{Z}_{a}(k+1)=\mathbf{H}_{a} \mathbf{X}_{a}(k+1)+\mathbf{V}_{a}(k+1)
\end{aligned}
$$

and

$$
\begin{aligned}
& \mathbf{X}_{j}(k+1)=\mathbf{F}_{j} \mathbf{X}_{j}(k)+\mathbf{W}_{j}(k) \\
& \mathbf{Z}_{j}(k+1)=\mathbf{H}_{j} \mathbf{X}_{j}(k+1)+\mathbf{V}_{j}(k+1)
\end{aligned}
$$

where

$$
\begin{aligned}
& \mathbf{X}_{a}=\left[\begin{array}{lllllllll}
x & \dot{x} & \ddot{x} & y & \mathrm{y} & \mathrm{y} & z & \dot{z} & \ddot{z}
\end{array}\right]^{T} \\
& \mathbf{X}_{j}=\left[\begin{array}{llllllllllll}
x & \dot{x} & \ddot{x} & \dddot{x} & y & \dot{y} & \ddot{y} & \dddot{y} & z & \dot{z} & \ddot{z} & \dddot{z}
\end{array}\right]^{T}
\end{aligned}
$$

and

$$
\begin{aligned}
& \mathbf{W}_{a}=\left[\begin{array}{lllllllll}
u_{1 a} & u_{2 a} & u_{3 a} & u_{4 a} & u_{5 a} & u_{6 a} & u_{7 a} & u_{8 a} & u_{9 a}
\end{array}\right]^{T} \\
& \mathbf{W}_{j}=\left[\begin{array}{lllllll}
u_{1 j} & u_{2 j} & u_{3 j} & u_{4 j} & u_{5 j} & u_{6 j} \\
u_{7 j} & u_{8 j} & u_{9 j} & u_{10 j} & u_{11 j} & u_{12 j}
\end{array}\right]^{2} .
\end{aligned}
$$


In (36) and (37) $u_{1 a}, u_{2 a}$, etc. are the driving noise components for the acceleration model, and $u_{1 j}, u_{2 j}$, etc. are those for the jerk model. Other symbols in (31) and (33) are

$$
\begin{aligned}
& \mathbf{H}_{a}=\left[\begin{array}{lllllllll}
1 & 0 & 0 & 0 & 0 & 0 & 0 & 0 & 0 \\
0 & 0 & 0 & 1 & 0 & 0 & 0 & 0 & 0 \\
0 & 0 & 0 & 0 & 0 & 0 & 1 & 0 & 0
\end{array}\right] \\
& \mathbf{H}_{j}=\left[\begin{array}{llllllllllll}
1 & 0 & 0 & 0 & 0 & 0 & 0 & 0 & 0 & 0 & 0 & 0 \\
0 & 0 & 0 & 0 & 1 & 0 & 0 & 0 & 0 & 0 & 0 & 0 \\
0 & 0 & 0 & 0 & 0 & 0 & 0 & 0 & 1 & 0 & 0 & 0
\end{array}\right] \\
& \mathbf{Z}_{a}=\left[\begin{array}{lll}
M_{x} & M_{y} & M_{z}
\end{array}\right]^{T} \\
& \mathbf{Z}_{j}=\left[\begin{array}{lll}
M_{x} & M_{y} & M_{z}
\end{array}\right]^{T}
\end{aligned}
$$

and

$\mathrm{V}_{a}=\left[\begin{array}{lll}\text { Noise in } M_{x} & \text { Noise in } M_{y} & \text { Noise in } M_{z}\end{array}\right]^{T}$

$\mathrm{V}_{j}=\left[\text { Noise in } M_{x} \quad \text { Noise in } M_{y} \quad \text { Noise in } M_{z}\right]^{T}$.

The measurements $R, \theta_{m}$, and $\varphi_{m}$ in spherical coordinates are transformed to Cartesian coordinates using

$$
\begin{aligned}
& M_{x}(k)=R_{m}(k) \cos \varphi_{m}(k) \cos \theta_{m}(k) \\
& M_{y}(k)=R_{m}(k) \cos \varphi_{m}(k) \sin \theta_{m}(k) \\
& M_{z}(k)=R_{m}(k) \sin \varphi_{m}(k) .
\end{aligned}
$$

Because of the transformation of measurements from spherical to Cartesian coordinates, the measurement noise covariance components in the Cartesian coordinates become correlated and are updated every iteration using the following relations:

$$
\begin{aligned}
& r_{11}= \sigma_{r}^{2} \cos ^{2} \varphi_{m}^{2}(k)+R_{m}^{2}(k) \\
& \times\left[\sigma_{\varphi}^{2} \sin \varphi_{m}^{2}(k) \cos \theta_{m}^{2}(k)\right. \\
&\left.+\sigma_{\theta}^{2} \cos \varphi_{m}^{2}(k) \sin \theta_{m}^{2}(k)\right] \\
& r_{22}= \sigma_{r}^{2} \cos \varphi_{m}^{2}(k) \sin \theta_{m}^{2}(k)+R_{m}^{2}(k) \\
& \times\left[\sigma_{\varphi}^{2} \sin \varphi_{m}^{2}(k) \sin \theta_{m}^{2}(k)\right. \\
&\left.+\sigma_{\theta}^{2} \cos \varphi_{m}^{2}(k) \cos \theta_{m}^{2}(k)\right] \\
& r_{33}= \sigma_{r}^{2} \sin \varphi_{m}^{2}(k)+R_{m}^{2}(k) \sigma_{\varphi}^{2} \cos \varphi_{m}^{2}(k) \\
& r_{12}= r_{21}=(1 / 2)\left\{\sigma_{r}^{2} \cos \varphi_{m}^{2}(k) \sin 2 \theta_{m}(k)\right. \\
&+R_{m}^{2}(k)\left[\sigma_{\varphi}^{2} \sin \varphi_{m}^{2}(k) \sin 2 \theta_{m}(k)\right. \\
&\left.\left.\quad \sigma_{\theta}^{2} \cos \varphi_{m}^{2}(k) \sin 2 \theta_{m}(k)\right]\right\} \\
& r_{13}= r_{31}=(1 / 2)\left\{\sigma_{r}^{2} \sin 2 \varphi_{m}(k) \cos \theta_{m}(k)\right. \\
&\left.-R_{m}^{2}(k) \sigma_{\varphi}^{2} \sin 2 \varphi_{m}(k) \cos \theta_{m}(k)\right\}
\end{aligned}
$$

and

$$
\begin{aligned}
r_{23}=r_{32}=(1 / 2)\{ & \sigma_{r}^{2} \sin 2 \varphi_{m}(k) \sin \theta_{m}(k) \\
& \left.-R_{m}^{2}(k) \sigma_{\varphi}^{2} \sin 2 \varphi_{m}(k) \sin \theta_{m}(k)\right\}
\end{aligned}
$$

where $\sigma_{r}^{2}, \sigma_{\theta}^{2}$, and $\sigma_{\varphi}^{2}$ denote the variances of the measurement noise in $r, \theta$, and $\varphi$ dimensions, respectively.

The state transition matrix for the acceleration filter is

$$
\mathbf{F}_{a}=\left|\begin{array}{ccccccccc}
1 & T & {\left[e^{-\alpha T}+\alpha T-1\right] / \alpha^{2}} & 0 & 0 & 0 & 0 & 0 & 0 \\
0 & 1 & {\left[1-e^{-\alpha T}\right] / \alpha} & 0 & 0 & 0 & 0 & 0 & 0 \\
0 & 0 & e^{-\alpha T} & 0 & 0 & 0 & 0 & 0 & 0 \\
0 & 0 & 0 & 1 & T & {\left[e^{-\alpha T}+\alpha T-1\right] / \alpha} & 0 & 0 & 0 \\
0 & 0 & 0 & 0 & 1 & {\left[1-e^{-\alpha T}\right] / \alpha} & 0 & 0 & 0 \\
0 & 0 & 0 & 0 & 0 & e^{-\alpha T} & 0 & 0 & 0 \\
0 & 0 & 0 & 0 & 0 & 0 & 1 & T & {\left[e^{-\alpha T}+\alpha T-1\right] / \alpha} \\
0 & 0 & 0 & 0 & 0 & 0 & 0 & 1 & {\left[1-e^{-\alpha T}\right] / \alpha} \\
0 & 0 & 0 & 0 & 0 & 0 & 0 & 0 & e^{-\alpha T}
\end{array}\right|
$$




$$
\mathbf{F}_{j}=\left[\begin{array}{cccccccccccc}
1 & T & T^{2} / 2 & p_{1} & 0 & 0 & 0 & 0 & 0 & 0 & 0 & 0 \\
0 & 1 & T & q_{1} & 0 & 0 & 0 & 0 & 0 & 0 & 0 & 0 \\
0 & 0 & 1 & r_{1} & 0 & 0 & 0 & 0 & 0 & 0 & 0 & 0 \\
0 & 0 & 0 & s_{1} & 0 & 0 & 0 & 0 & 0 & 0 & 0 & 0 \\
0 & 0 & 0 & 0 & 1 & T & T^{2} / 2 & p_{1} & 0 & 0 & 0 & 0 \\
0 & 0 & 0 & 0 & 0 & 1 & T & q_{1} & 0 & 0 & 0 & 0 \\
0 & 0 & 0 & 0 & 0 & 0 & 1 & r_{1} & 0 & 0 & 0 & 0 \\
0 & 0 & 0 & 0 & 0 & 0 & 0 & s_{1} & 0 & 0 & 0 & 0 \\
0 & 0 & 0 & 0 & 0 & 0 & 0 & 0 & 1 & T & T^{2} / 2 & p_{1} \\
0 & 0 & 0 & 0 & 0 & 0 & 0 & 0 & 0 & 1 & T & q_{1} \\
0 & 0 & 0 & 0 & 0 & 0 & 0 & 0 & 0 & 0 & 1 & r_{1} \\
0 & 0 & 0 & 0 & 0 & 0 & 0 & 0 & 0 & 0 & 0 & s_{1}
\end{array}\right]
$$

where the elements of the symmetric matrix are

$$
\begin{aligned}
q_{11}^{\prime}=\left(1 / 2 \alpha^{5}\right)\left[1-e^{-2 \alpha T}+2 \alpha T+2 \alpha^{3} T^{3} / 3\right. \\
\left.-2 \alpha^{2} T^{2}-4 \alpha T e^{-\alpha T}\right] \\
q_{12}^{\prime}=\left(1 / 2 \alpha^{4}\right)\left(e^{-2 \alpha T}+1-2 e^{-\alpha T}-2 \alpha T+\alpha^{2} T^{2}\right) \\
q_{13}^{\prime}=\left(1 / 2 \alpha^{3}\right)\left(1-e^{-2 \alpha T}-2 \alpha T e^{-\alpha T}\right) \\
q_{22}^{\prime}=\left(1 / 2 \alpha^{3}\right)\left(4 e^{-\alpha T}-3-e^{-2 \alpha T}+2 \mathrm{aT}\right) \\
q_{23}^{\prime}=\left(1 / 2 \alpha^{2}\right)\left(e^{-2 \alpha T}+1-2 e^{-\alpha T}\right)
\end{aligned}
$$

and

$$
q_{33}^{\prime}=(1 / 2 \alpha)\left(1-e^{-2 \alpha T}\right)
$$

Similarly, the process noise covariance for the jerk model is

$$
\mathbf{Q}_{j}(k)=2 \alpha \sigma_{j}^{2}\left|\begin{array}{cccccccccccc}
q_{11} & q_{12} & q_{13} & q_{14} & 0 & 0 & 0 & 0 & 0 & 0 & 0 & 0 \\
q_{21} & q_{22} & q_{23} & q_{24} & 0 & 0 & 0 & 0 & 0 & 0 & 0 & 0 \\
q_{31} & q_{32} & q_{33} & q_{34} & 0 & 0 & 0 & 0 & 0 & 0 & 0 & 0 \\
q_{41} & q_{42} & q_{43} & q_{44} & 0 & 0 & 0 & 0 & 0 & 0 & 0 & 0 \\
0 & 0 & 0 & 0 & q_{11} & q_{12} & q_{13} & q_{14} & 0 & 0 & 0 & 0 \\
0 & 0 & 0 & 0 & q_{21} & q_{22} & q_{23} & q_{24} & 0 & 0 & 0 & 0 \\
0 & 0 & 0 & 0 & q_{31} & q_{32} & q_{33} & q_{34} & 0 & 0 & 0 & 0 \\
0 & 0 & 0 & 0 & q_{41} & q_{42} & q_{43} & q_{44} & 0 & 0 & 0 & 0 \\
0 & 0 & 0 & 0 & 0 & 0 & 0 & 0 & q_{11} & q_{12} & q_{13} & q_{14} \\
0 & 0 & 0 & 0 & 0 & 0 & 0 & 0 & q_{21} & q_{22} & q_{23} & q_{24} \\
0 & 0 & 0 & 0 & 0 & 0 & 0 & 0 & q_{31} & q_{32} & q_{33} & q_{34} \\
0 & 0 & 0 & 0 & 0 & 0 & 0 & 0 & q_{41} & q_{42} & q_{43} & q_{44}
\end{array}\right|
$$

The noise covariance matrix for the acceleration model is

$$
\mathbf{Q}_{a}(k)=2 \alpha \sigma_{m}^{2}\left[\begin{array}{ccccccccc}
q_{11}^{\prime} & q_{12}^{\prime} & q_{13}^{\prime} & 0 & 0 & 0 & 0 & 0 & 0 \\
q_{21}^{\prime} & q_{2 \mathbf{z}}^{\prime} & q_{33}^{\prime} & 0 & 0 & 0 & 0 & 0 & 0 \\
q_{31}^{\prime} & q_{32}^{\prime} & q_{33}^{\prime} & 0 & 0 & 0 & 0 & 0 & 0 \\
0 & 0 & 0 & q_{11}^{\prime} & q_{12}^{\prime} & q_{13}^{\prime} & 0 & 0 & 0 \\
0 & 0 & 0 & q_{21}^{\prime} & q_{22}^{\prime} & q_{23}^{\prime} & 0 & 0 & 0 \\
0 & 0 & 0 & q_{31}^{\prime} & q_{32}^{\prime} & q_{33}^{\prime} & 0 & 0 & 0 \\
0 & 0 & 0 & 0 & 0 & 0 & q_{11}^{\prime} & q_{12}^{\prime} & q_{13}^{\prime} \\
0 & 0 & 0 & 0 & 0 & 0 & q_{21}^{\prime} & q_{22}^{\prime} & q_{23}^{\prime} \\
0 & 0 & 0 & 0 & 0 & 0 & q_{31}^{\prime} & q_{32}^{\prime} & q_{33}^{\prime}
\end{array}\right]
$$

where the elements have the same expression as defined in (20) for the one-dimensional case. In (48) and (49), Q is the correlation parameter for acceleration or jerk depending on the model,

The derivation of the jerk model for tracking in three dimensions is now complete, along with an acceleration model for comparison.

\section{SIMULATION RESULTS AND DISCUSSION}

The enhancement of tracking performance in three-dimensional space by the use of the jerk model with a 4-state filter over the acceleration model used with a 3-state filter is illustrated here through numerical simulation. Fig. 1 shows the kinematic 

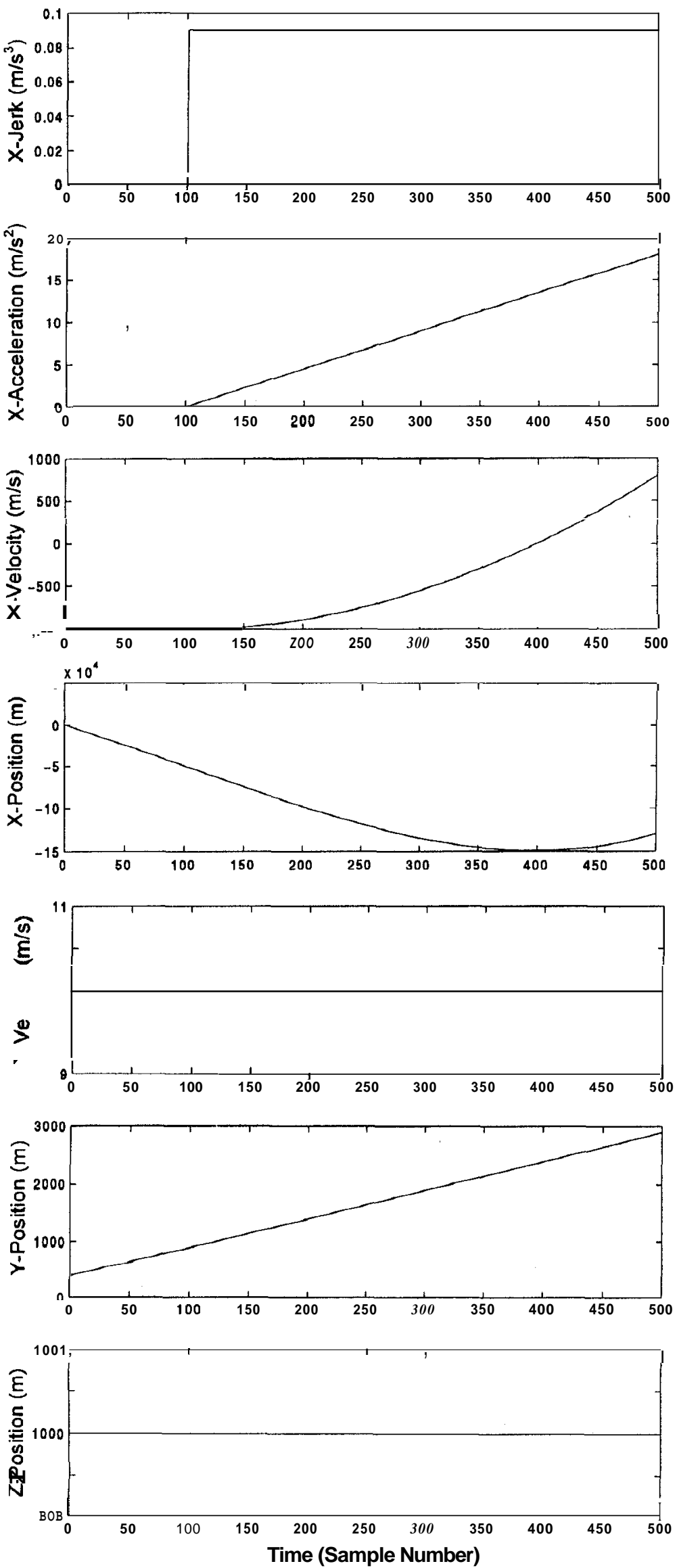

Fig. 1. Target maneuver parameters as functions of time. 

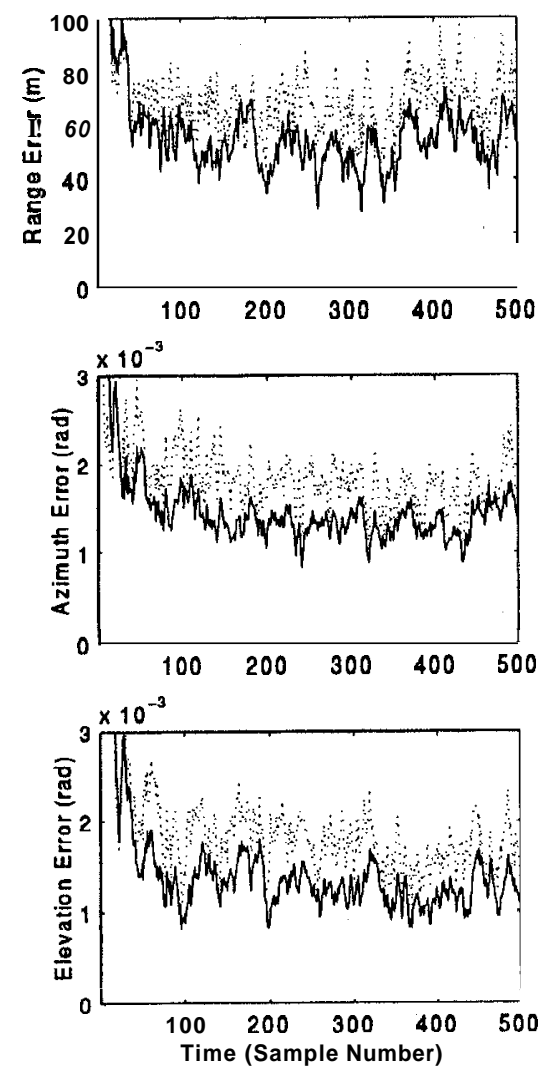

Fig. 2. Evolution of tracking errors in range, azimuth, and elevation. Solid lines show jerk filter behavior and dots show acceleration filter behavior.

parameters of the target as functions of time (i.e., sample number, with sample interval $T=0.5 \mathrm{~s}$ ). Along the $x$ axis, the target starts with a constant velocity of $-1000 \mathrm{~m} / \mathrm{s}$, and a step jerk of $0.09 \mathrm{~m} / \mathrm{s}^{3}$ is applied at $50 \mathrm{~s}$ (100th sample), resulting in a ramp acceleration, parabolic velocity variation, and cubic position variation. The y-velocity component is kept constant at $10 \mathrm{~m} / \mathrm{s}$, and the $\mathrm{z}$ position is held constant at $1000 \mathrm{~m}$. The following statistical parameter values are chosen for the simulation.

Measurement noise covariance

$$
=\left\{\begin{array}{l}
22,500 \mathrm{~m}^{2} \text { (range) } \\
25 \times 10^{-6} \mathrm{rad}^{2} \text { (azimuth). } \\
25 \times 10^{-6} \mathrm{rad}^{2} \text { (elevation) }
\end{array}\right.
$$

Correlation parameter $\alpha=0.006$ (for both acceleration and jerk models).

Process noise variance for acceleration model, $Q_{a}=2 \alpha \sigma \hat{m}, \sigma_{m}=18 \mathrm{~m} / \mathrm{s}^{2}$.

Process noise variance for jerk model, $Q_{j}=2 \alpha \sigma_{j}^{2}$, $\sigma_{j}=0.09 \mathrm{~m} / \mathrm{s}^{3}$.

It is to be noted here that a common value of the correlation parameter $a$ has been chosen for both the models. The two motion models are physically different in the sense that in one case the target acceleration is correlated while in the other it is
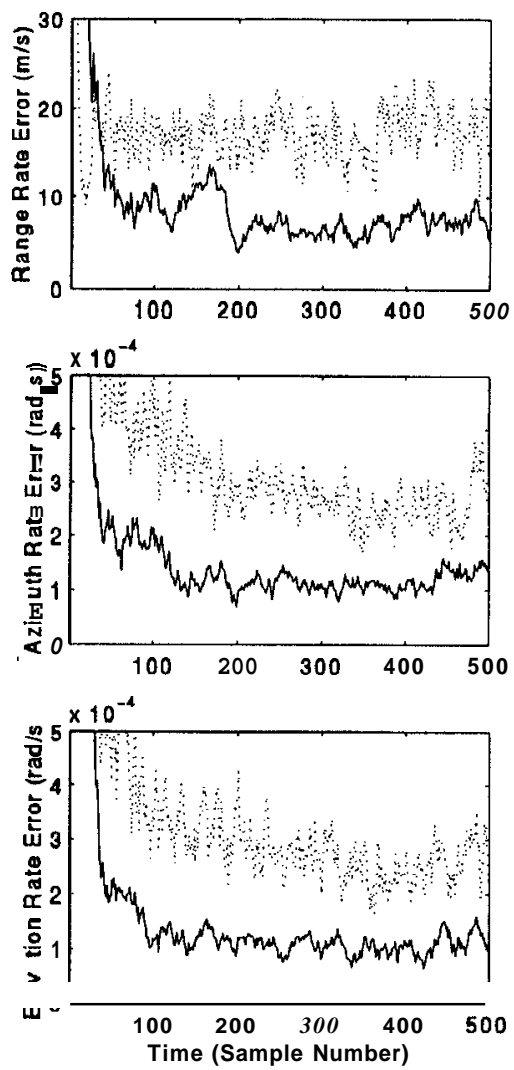

Fig. 3. Evolution of tracking errors in rates of range, azimuth, and elevation. Solid lines show jerk filter behavior and dots show acceleration filter behavior.

the target jerk that is correlated. Thus, using these two models it is not possible to describe a common physical type of target motion when $\alpha$ is the only control variable in each model. The best that can be done, therefore, is to maintain a degree of analytical similarity by assuming a common $a$, and using a common target trajectory for comparing the two tracking filters, as done here.

The three-dimensional jerk filter is initialized in each of the three orthogonal axes in a manner similar to the one-dimensional case discussed earlier, using the first three measurements. Thus, equations analogous to (22) are written for the $y$ and $z$ axes also. The $\mathbf{P}$ matrix is now $12 \times 12$, consisting of three diagonal submatrices, each of which is similar to the $4 \times 4 \mathbf{P}$ matrix for the one-dimensional case. The small value of a permits the use of the simpler form (29) here for each of the submatrices. An analogous procedure is employed to initialize the acceleration filter using the first two measurements.

Following initialization, the matrices (46) and (47) are used for updating states (prediction) in the acceleration and jerk models, respectively. Next, the predicted covariance and Kalman gain matrices are found using standard Kalman filter equations. The measurements are simulated by adding zero-mean Gaussian random numbers of known covariances to the $r, \theta, \varphi$ values at corresponding points in the 

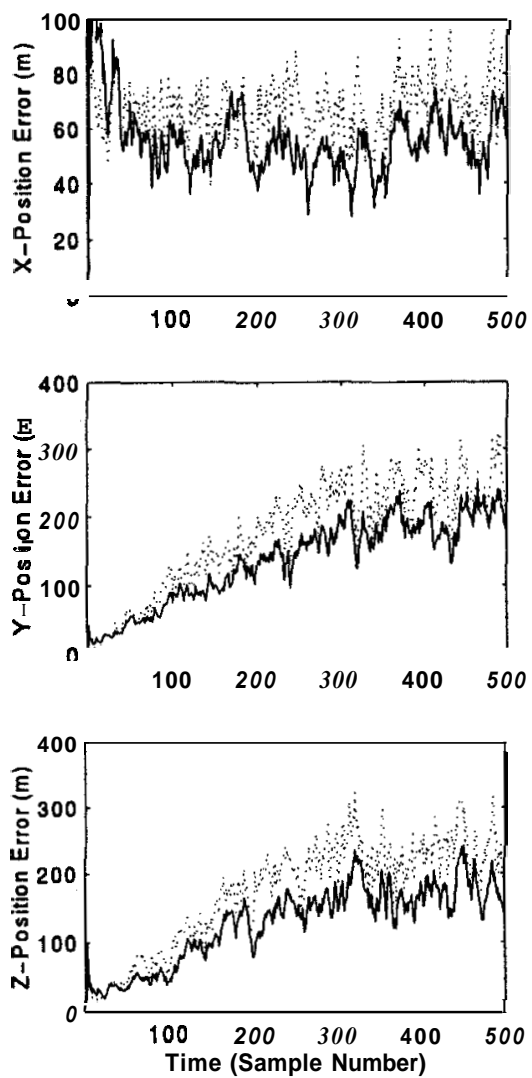

Fig. 4. Evolution of tracking errors in $x, y$, and $z$ components of target position. Solid lines show jerk filter behavior and dots show acceleration filter behavior.

trajectory, and these are transformed to the Cartesian frame using (44).

The results of simulation are shown in Figs. 2-6. The plots in these figures are the rms value of 20 random runs, with the same set of random numbers used in each case. In Fig. 2 are shown the errors in range, azimuth, and elevation for both jerk and acceleration models. The errors in range rate, azimuth rate, and elevation rate are shown in the plots of Fig. 3. Fig. 4 shows the errors in the Cartesian position variables $\boldsymbol{x}, \boldsymbol{y}$, and $z$. To conserve space, the errors in velocity estimates along the $\boldsymbol{x}$ and $y$ coordinates only are plotted in Fig. 5, and the acceleration errors along these axes are shown in Fig. 6. It is clear from the simulation results that the jerk model provides superior tracking performance compared with the acceleration model in respect of all the tracking variables. The margin of improvement is modest but clear for position variables themselves (in both polar and Cartesian coordinates), and increases with the order of the derivatives of position. As seen from Fig. 6, the steady-state errors in the acceleration estimates are much higher for the acceleration model than for the jerk model.

It is to be noted that a relatively slow maneuver (as indicated by the highest acceleration value) has been used in the simulation example shown in this
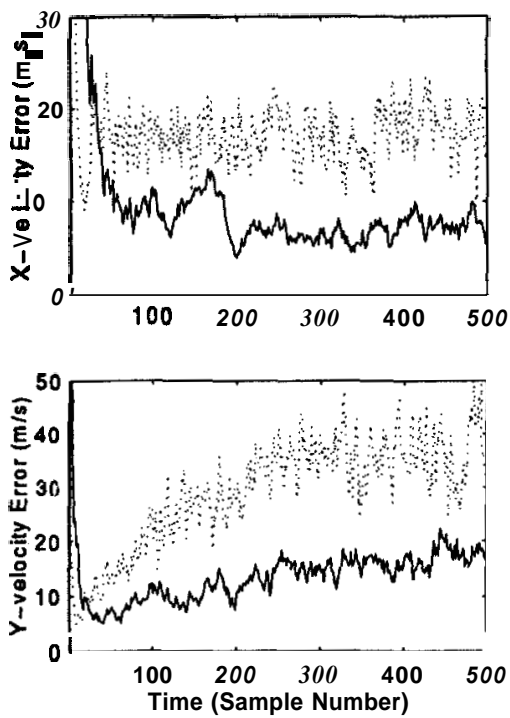

Fig. 5. Evolution of tracking errors in velocities along $x$ and $y$ directions. Solid lines show jerk filter behavior and dots show acceleration filter behavior.
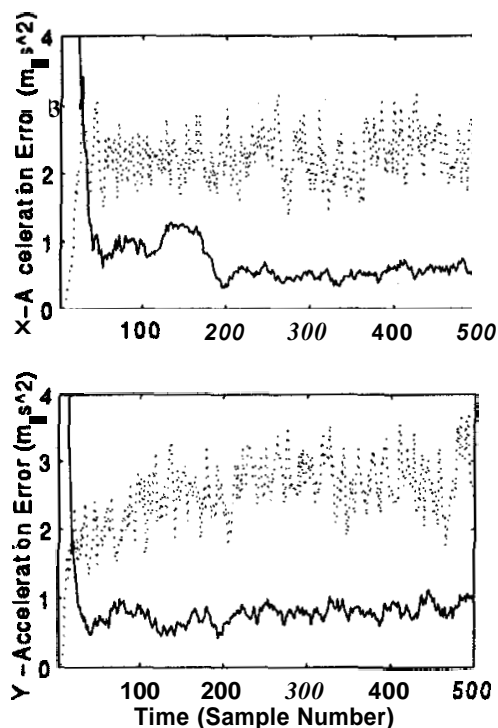

Fig. 6. Evolution of tracking errors in accelerations along $x$ and $y$ directions. Solid lines show jerk filter behavior and dots show acceleration filter behavior.

section. Even for such a target, the jerk filter comes out much better than the acceleration filter, primarily because of the presence of jerk in the maneuver. We have experimented with more vigorous maneuvers, for which the jerk filter still provides good tracking behavior while the acceleration filter fails to converge and/or develops large biases. These problems may be obviated by using very large values of $Q$, but this leads to large variance in the estimates.

Based on the limited simulation reported here, it cannot be claimed that the jerk filter is better than the acceleration filter under all conditions. However, in generalized maneuvers such as dog-fights, where the target acceleration is not necessarily constant, jerk will 

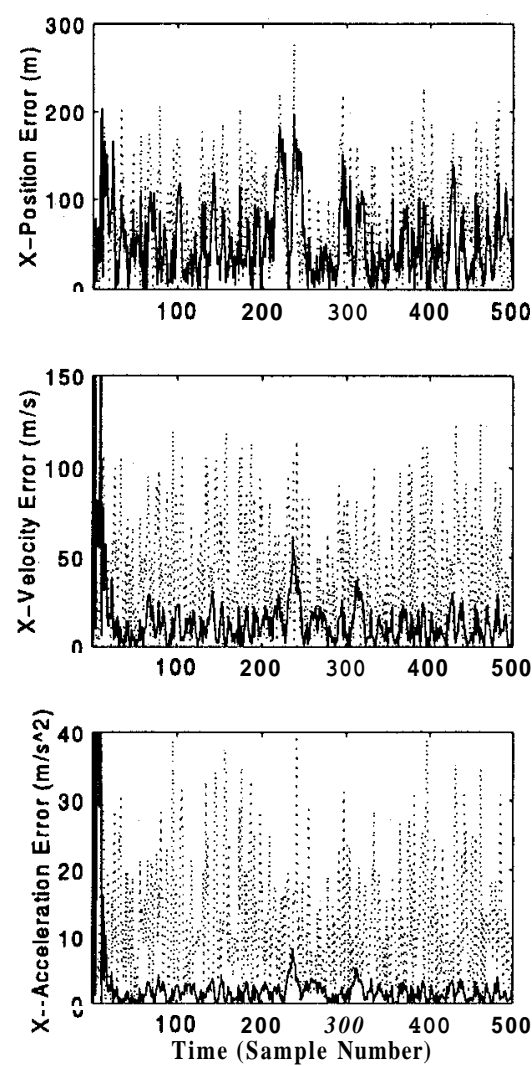

Fig. 7. Evolution of tracking error in the position, velocity, and acceleration along $x$ direction under model-filter mismatch condition. Solid lines show jerk filter behavior and dots show acceleration filter behavior.

most often be present, and it will be fair to expect the jerk filter to provide a higher tracking accuracy than the acceleration filter. As the example in this section shows, this is true even when the jerk and acceleration values are rather low. With higher jerk levels in the maneuver the advantage of the jerk filter will be even more strikingly apparent.

\section{EFFECT OF MODEL-FILTER MISMATCH}

Sophisticated tracking filters often suffer from the drawback that their performance degrades significantly when the actual target maneuver departs from the assumed model for the maneuver. Such a situation is known as model-filter mismatch or plant-filter mismatch. It is therefore necessary to comment on the robustness of the jerk filter, presented in this work, with respect to such mismatch. A full study of the mismatch phenomenon in all its aspects is beyond the scope of this work due to length constraints. However, results concerning one important type of mismatch are presented in Fig. 7 (only the $x$ axis variables are shown for brevity) wherein the value of the correlation parameter $a$ is assumed to be widely different between the maneuver model and the tracking filter. Thus, against of the assumed value of 0.006 for $a$ the actual value of this parameter is set at a much higher value of 0.6. Even with such a high level of mismatch, Fig. 7 shows that the jerk filter continues to perform significantly better than the acceleration filter.

\section{SUMMARY}

A higher order model for target tracking in three dimensions than what is available hitherto has been presented. It consists of a jerk model of target motion, and a tracking filter of compatible order. The model and filter structures have been explicitly derived, and the initialization process clearly enunciated.

The motivation for introducing a higher order model is that more agile target maneuvers are likely to have more significant higher order derivatives which a lower order tracking model, such as the velocity or acceleration models currently in use, cannot adequately handle. The premise that the jerk model can track more nimble maneuvers better is validated by numerical simulations, of which one example has been given in the paper. The jerk filter shows much better performance than the acceleration filter when both are able to track the target, and the jerk filter continues to track well in cases of high target maneuver where the acceleration filter fails.

\section{ACKNOWLEDGMENT}

The authors gratefully acknowledge the numerous discussions with their colleague, Prof. M. R. Ananthasayanam, which lent clarity to some of the ideas. They also thank Mr. S. Roychoudhury, General Manager, HAL, Hyderabad, for his constant support and practical suggestions.

\section{REFERENCES}

[1] Schooler, C. C. (1975) Optimal alpha beta filters for systems with modeling inaccuracies.

IEEE Transactions on Aerospace and Electronic Systems, AES-11 (Nov. 1975), 1300-1306.

[2] Kalata, P. R. (1984)

The tracking index: A generalized parameter for alpha beta target trackers.

IEEE Transactions on Aerospace and Electronic Systems, AES-LO (Mar. 1984), 174-182.

[3] Solomon, D. L. (1985)

Covariance matrix for alpha, beta, gamma filtering. IEEE Transactions on Aerospace and Electronic Systems, AES-21 (Jan. 1985), 157-159.

[4] Thorp, J. S. (1973)

Optimal tracking of maneuvering targets.

IEEE Transactions on Aerospace and Electronic Systems, AES-9 (July 1973), 512-519.

[5] Singer, R. A. (1970)

Estimating optimal tracking filter performance for manned maneuvering targets.

IEEE Transactions on Aerospace and Electronic Systems, AES-6 (July 1970), 473-483. 
[6] Spingarn, K., and Weidemann, H. L. (1972)

Linear regression filtering and prediction for tracking maneuvering aircraft targets.

IEEE Transactions on Aerospace and Electronic Systems, AES-8 (Nov. 1972), 800-810.

[7] Moose, R. L. (1975)

An adaptive state estimation solution to the maneuvering target problem.

IEEE Transactions on Automutic Control, AC-20 (June 1975), 359-362.

[8] Moose, R. L., Vanlandingham, H. F., and McCabe, D. H. (1979)

Modeling and estimation for tracking maneuvering targets.

IEEE Transactions on Aerospace and Electronic Systems, AES-15 (May 1979), 448-456.
[9] Bar-Shalom, Y., and Birmiwal, K. (1982)

Variable dimension filter for maneuvering target tracking. IEEE Transactions on Aerospace and Electronic Systems, AES-18 (Sept. 1982), 621-629.

[10] Sudano, J. J. (1993)

The $\alpha-\beta-\Gamma$ tracking filter with a noisy jerk as the maneuver model.

IEEE Transactions on Aerospace and Electronic Systems, 29 (Apr. 1993), 578-580.

[11] Hoffman, S. A., and Blair, W. D. (1994)

Comments on "The $\alpha-\beta-\Gamma$ tracking filter with a noisy jerk as the maneuver model".

IEEE Transactions on Aerospace and Electronic Systems, 30 (July 1994), 925-928.
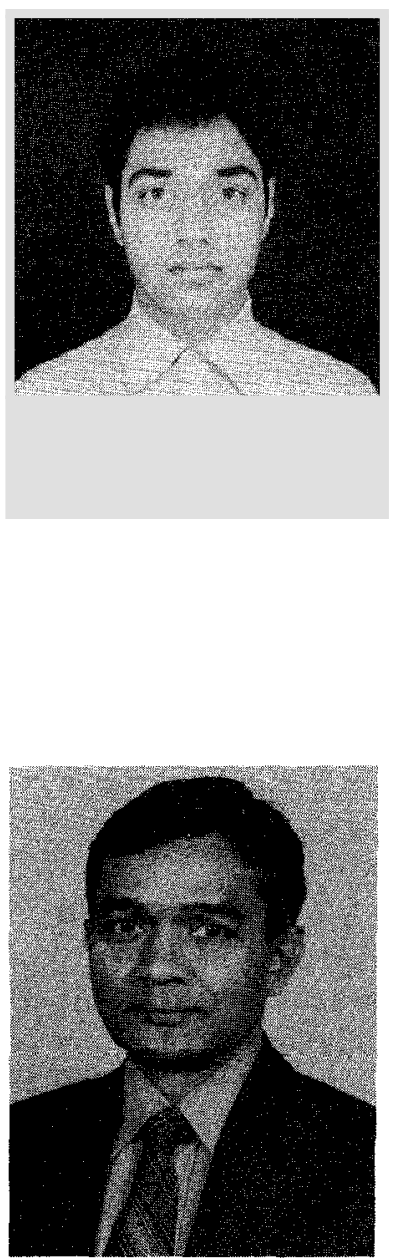

Kishore Mehrotra was born at Lucknow, India on Feb. 17, 1958. He received the B.Tech. degree in electrical engineering from the Indian Institute of Technology, Kanpur in 1979, and the Ph.D. from the Indian Institute of Science, Bangalore in 1994.

From 1979 to 1996 he worked in the Avionics Design Bureau of Hindustan Aeronautics Limited, Hyderabad, India, where, since 1989, he held the position of Manager (Designs). He is presently working as a Design Engineer at Swichtec Power Systems, Christchurch, New Zealand. His research interests are in the areas of radar tracking, digital signal processing, power electronics, control systems, and mathematical modeling.

Pravas R. Mahapatra received the B.Sc. (Engg.) degree with Honors from the Regional Engineering College at Rourkela, India, and the M.E. (with Distinction) and $\mathrm{Ph} . \mathrm{D}$. degrees from the Indian Institute of Science at Bangalore, India.

Since 1970 he has been teaching at the Department of Aerospace Engineering of the Indian Institute of Science where he is currently a professor. On sabbatical leave, he has held Regular and Senior Research Associateships of the U.S. National Research Council, and has been a Visiting Scientist at the University of Oklahoma. He has a broad area of research interest within the field of aerospace and electronic systems including radar systems, navigation theory, electronic navigational aids, aviation safety problems with particular reference to weather phenomena and air traffic control, and aspects of signal processing.

Dr. Mahapatra has authored or co-authored over one hundred scientific papers, most of which have appeared in international publications. He is a Fellow of the Institution of Electronics and Telecommunication Engineers (India) and is a Professional Member of the Institute of Navigation (U.S.A.).He has received several awards, including the 1993 IEEE Donald G. Fink Prize. 